\title{
Caracterização dos profissionais intensivistas em unidade de internação de pacientes com COVID-19
}

Characterization of intensive care professionals in an inpatient unit of patients with COVID-19

Caracterización de profesionales de cuidados intensivos en una unidad de hospitalización de pacientes con COVID-19

Pedro Elias Souza ${ }^{1 *}$, Rosane Dias Rosa ${ }^{1}$, Claudete Barbosa Ruschival ${ }^{1}$, João Gabriel Linhares Pulner $^{1}$, Mônica de Cassia Lôbo Souto Maior ${ }^{1}$, Fernando Luiz Westphal ${ }^{1}$.

\section{RESUMO}

Objetivo: Descrever o perfil epidemiológico e características do trabalho de profissionais atuantes em UTI de pacientes com COVID-19, seus principais meios de atualização frente à pandemia e fatores de estresse a que são submetidos. Métodos: Trata-se de estudo transversal, envolvendo profissionais atuantes em UTICOVID-19, aprovado pelo Comitê de Ética em Pesquisa e realizado em agosto de 2020, com dados coletados por meio da plataforma Google Forms. Resultados: Foram avaliados 63 profissionais, 41,3\% técnicos de enfermagem e $27 \%$ enfermeiros; $52 \%$ com menos de 40 anos e com média de 13 anos de atuação profissional. A Internet foi utilizada por $96,8 \%$ dos avaliados para sanar dúvidas profissionais. A carga horária diária de $77,7 \%$ dos profissionais foi de 12 horas e a realização de curativos e banhos no leito foram consideradas as atividades mais demoradas e cansativas; $46 \%$ dos profissionais foram acometidos por COVID-19. Conclusão: Profissionais atuantes em UTI COVID eram, majoritariamente, da área da enfermagem e com menos de 40 anos. A alta taxa de infecção por COVID na equipe, demora e cansaço na realização de determinadas atividades e o regime de trabalho podem ser considerados fatores estressores na equipe. A Internet se mostrou o principal meio de atualização utilizado.

Palavras-chave: Unidades de terapia intensiva, Esgotamento psicológico, COVID-19.

\section{ABSTRACT}

Objective: To describe the epidemiological profile and characteristics of the work of professionals working in the ICU of patients with COVID-19, their main means of updating the pandemic and stress factors to which they are submitted. Methods: This is a cross-sectional study involving professionals working in ICU-COVID19, approved by the Research Ethics Committee, and conducted in August 2020, with data collected through the Google Forms platform. Results: We evaluated 63 professionals, $41.3 \%$ nursing technicians and $27 \%$ nurses; $52 \%$ under 40 years of age and with an average of 13 years of professional experience. The Internet was used by $96.8 \%$ of the evaluated to answer professional doubts. The daily workload of $77.7 \%$ of the professionals was 12 hours and the performance of dressings and baths in the bed were considered the most time-consuming and tiring activities. $46 \%$ of the professionals were affected by COVID-19. Conclusion: Professionals working in ICU COVID were mostly in the nursing area and under 40 years of age. The high rate of COVID infection in the team, delay, and tiredness in performing certain activities and the work regimen can be considered stressors in the team. The Internet proved to be the main means of updating used.

Keywords: Intensive care units, Burnout, Psychological, COVID-19.

\section{RESUMEN}

Objetivo: Describir el perfil epidemiológico y las características del trabajo de los profesionales que trabajan en la UCI de pacientes con COVID-19, sus principales medios de actualización de la pandemia y los factores de estrés a los que están sometidos. Métodos: Se trata de un estudio transversal en el que participan profesionales que trabajan en UCI-COVID-19, aprobado por el Comité de Ética en Investigación y realizado en agosto de 2020, con datos recopilados a través de la plataforma Google Forms. Resultados: Se evaluaron 63 profesionales, $41,3 \%$ técnicos de enfermería y $27 \%$ enfermeros; $52 \%$ menores de 40 años

1 Universidade Federal do Amazonas (UFAM), Manaus - AM. *E-mail: pedroeliassouza@ufam.edu.br Estudo financiado pela Fundação de Amparo a Pesquisa do Estado do Amazonas (FAPEAM). SUBMETIDO EM: 7/2021 
y con una media de 13 años de experiencia profesional. Internet fue utilizado por el 96,8\% de los evaluados para responder dudas profesionales. La carga de trabajo diaria del 77,7\% de los profesionales fue de 12 horas y la realización de apósitos y baños en la cama fueron consideradas las actividades más lentas y agotadoras. El $46 \%$ de los profesionales se vieron afectados por el COVID-19. Conclusión: Los profesionales que trabajan en UCI COVID se encontraban en su mayoría en el área de enfermería y menores de 40 años. La alta tasa de infección por COVID en el equipo, el retraso y el cansancio en la realización de ciertas actividades y el régimen de trabajo pueden considerarse factores estresantes en el equipo. Internet demostró ser el principal medio de actualización utilizado.

Palabras clave: Unidades de cuidados intensivos, Agotamiento psicológico, COVID-19.

\section{INTRODUÇÃO}

As Unidades de Terapia Intensiva (UTIs) são ambientes intra-hospitalares reservados para acompanhamento de pacientes críticos que necessitam de cuidado especializado, contínuo e multiprofissional (ALVES FAD, et al., 2020). Permitem a monitorização contínua, invasiva ou não, de pacientes por meio de tecnologia especializada (HASSEGAWA LCU, et al., 2017). De acordo com sua complexidade e nível de cuidado são determinadas as responsabilidades técnicas, éticas, habilitações e atribuições da equipe necessária para seu adequado funcionamento (CONSELHO FEDERAL DE MEDICINA, 2020).

De acordo com o Ministério da Saúde, em dezembro de 2020 o Brasil possuía 19.544 leitos de UTI reservados para pacientes infectados com o vírus Sars-CoV-2 (portadores da COVID-19) (BRASIL, 2020). Em março de 2021, o Brasil apresentou mais de 11 milhões de pacientes acometidos por COVID-19 e cerca de 310 mil mortes decorrentes da infecção (WORLD HEALTH ORGANIZATION, 2021). Do total de acometidos, entre 5\% e 10\% apresentam a forma grave da doença (insuficiência respiratória aguda grave), necessitando seguimento em leitos de UTI, justificando a reserva de tais vagas (SALLES NETO LL, et al., 2020).

Além das infecções e mortes, a COVID-19 influenciou na saúde mental da população, aumentando os níveis de estresse, ansiedade, depressão e medo (YILDIRIM M e SOLMAZ F, 2020; ARSLAN G, et al., 2020). Os impactos na saúde mental podem ser explicados a partir do estado de quarentena enfrentado pela população, com seus efeitos psicológicos negativos (BROOKS SK, et al., 2020). Apesar de que, as alterações na rotina diária, associadas à incerteza da duração desse período, bem como o medo de infecção, frustração, suprimentos inadequados, perda financeira e estigma, também podem justificar os problemas psicológicos (YILDIRIM M e SOLMAZ F, 2020; ARSLAN G, et al., 2020; BROOKS SK, et al., 2020).

No que se refere à população de profissionais de saúde, observou-se aumento no nível de estresse, ansiedade e Burnout durante a pandemia COVID-19 (MORGANTINI LA, et al., 2020). Antes mesmo do início da pandemia, problemas psicológicos relacionados ao estresse, ansiedade e síndrome de Burnout já eram descritos entre profissionais de saúde de UTIs (MARQUES GLC, et al., 2018; SILVA AF e ROBAZZI MLCC, 2019).

Burnout é uma síndrome psicológica que causa exaustão emocional, despersonalização e redução do sentimento de realização profissional, provocada principalmente por estresse interpessoal, especialmente no ambiente de trabalho (MASLACH C e LEITER MP, 2016).

A ansiedade, por sua vez, é caracterizada por preocupação excessiva relacionada às atividades cotidianas (MOURA A, et al., 2018). Pode ocorrer de forma adaptativa (decorrente de adversidades) ou como um transtorno psicológico, e a diferenciação entre estas formas decorre do tempo e severidade do acometimento (MARTINS BG, et al., 2019).

A fadiga por compaixão, caracterizada por esgotamento emocional e físico é, também, comumente descrita entre os profissionais de saúde atuantes em hospitais. Sua origem decorre de fatores laborais e individuais a partir da relação de empatia com o sofrimento de outro. Geralmente está associada à síndrome de Burnout, performando uma queda na qualidade de vida dos acometidos (TORRES JDRV, et al., 2018). 
Por tratar-se de uma pandemia sem precedentes, há muitos questionamentos acerca dos procedimentos a serem adotados no enfrentamento da doença, o que demanda um esforço redobrado em termos de capacitação e educação permanente desses profissionais (TEIXEIRA CFS, et al., 2020).

A pandemia da doença coronavírus é o maior experimento natural de todos os tempos, que vêm sendo testemunhado pelo mundo. Estudos científicos que possam elucidar aspectos deste evento são essenciais, neste momento (FORTALEZA CMCB, 2020). Desse modo, o presente artigo teve como objetivo identificar e descrever o perfil epidemiológico de profissionais de saúde atuantes em UTI de pacientes com COVID-19, seus principais meios de atualização frente à pandemia, e fatores de estresse a que são submetidos no desempenho de suas atividades.

\section{MÉTODOS}

Trata-se de um estudo transversal, observacional do tipo descritivo exploratório, com abordagem quantitativa, envolvendo a população de profissionais de saúde atuantes em UTI de pacientes acometidos por COVID-19 (denominada UTI COVID), de um Hospital Universitário do estado do Amazonas. A pesquisa foi realizada no período entre os dias 17 e 21 de agosto de 2020.

A amostra foi constituída de profissionais médicos, enfermeiros, fisioterapeutas, técnicos de enfermagem e técnicos de hemodiálise, fisioterapeuta, psicólogo, ou outro profissional atuando na UTI. Foram incluídos todos aqueles que concordaram em participar do estudo, mediante assinatura do Termo de Consentimento Livre e Esclarecido (TCLE) e excluídas profissionais grávidas e os que não manifestaram interesse em participar da pesquisa.

Para a coleta de dados, foi elaborado um questionário que foi armazenado na plataforma Google Forms, que foi escolhida por sua facilidade em compartilhar formulários com o público da amostra, ser online, gratuita e apresentar feedback instantâneo por meio de análises dos dados e sua apresentação em planilhas. O questionário foi compartilhado com os participantes de forma remota, utilizando-se da internet por meio de aplicativos de mensagens por celular e e-mails.

O formulário foi composto por perguntas objetivas, abordando aspectos sociodemográficos e relativos à atividade profissional dos participantes, como idade, escolaridade, profissão, tempo de atuação na área, carga horária diária e quantidade de plantões semanais, atividade mais e menos cansativa desenvolvida, e atividade mais e menos demorada desenvolvida; uso da Internet para aprendizado e sua importância para a atuação profissional; grau de importância das medidas de proteção individuais e informação mais importante para a segurança individual contra infecção por COVID.

No tratamento estatístico, as variáveis quantitativas foram descritas na forma de média e desvio padrão e as categóricas, por números absolutos e relativos.

O projeto foi submetido ao Comitê de Ética em Pesquisa (CEP) da Universidade Federal do Amazonas, com o número de Certificado de Apresentação para Apreciação Ética - CAAE: 33531420.1.0000.520, parecer 4.195.111. O TCLE foi preparado de acordo com a resolução $\mathrm{N}^{\circ} 510$, de 7 de abril de 2016 do Conselho Nacional de Saúde, e foram cumpridos os princípios éticos nela contidos.

\section{RESULTADOS}

A amostra foi constituída por 63 profissionais, mais frequentemente com idade entre 31 e 40 anos $(38,1 \%)$, com nível superior completo $(84,1 \%)$, distribuídos, de forma majoritária, nas atividades de enfermagem, com $41,3 \%$ atuando como técnicos de enfermagem e $27,0 \%$, como enfermeiros. Os profissionais com menor frequência na equipe foram a nutricionista e a psicóloga, 1,6\% cada.

Quanto à forma e fonte de atualização dos conhecimentos na área de atuação, a maioria utiliza, diariamente $(63,5 \%)$, a internet para pesquisas $(96,8 \%)$.

Indagados, a partir de uma escala de cinco pontos, sobre a importância da internet no trabalho, a maioria considerou o nível cinco (65,1\%) (maior nível de importância) e 6,3\% conferiram nível um (menor nível de importância) à Internet. 
Materiais na Internet, como vídeos, artigos, estudos, protocolos, entre outros, também foram citados como os meios mais utilizados na busca da atualização profissional (82,5\%). A utilização de vídeos informativos, a partir da plataforma Youtube, foi citada como a principal forma de utilização da rede, sendo empregada por $88,9 \%$ dos avaliados (Tabela 1 ).

Tabela 1 - Distribuição das caraterísticas sociodemográficas, profissionais e de busca por atualização na área de atuação, de intensivistas atuantes em UTI COVID, de Hospital Universitário, de 17 a 21 de agosto de $2020(n=63)$.

\begin{tabular}{|c|c|c|}
\hline Dados sociodemográficos & $\mathbf{n}$ & $\%$ \\
\hline \multicolumn{3}{|l|}{ Faixa etária (anos) } \\
\hline 21 a 30 & 9 & 14,3 \\
\hline 31 a 40 & 24 & 38,1 \\
\hline 41 a 50 & 13 & 20,6 \\
\hline 51 a 60 & 17 & 27,0 \\
\hline \multicolumn{3}{|l|}{ Nível de instrução } \\
\hline Superior completo & 53 & 84,1 \\
\hline Superior incompleto & 1 & 1,6 \\
\hline Médio & 9 & 14,3 \\
\hline \multicolumn{3}{|l|}{ Formação acadêmica } \\
\hline Enfermeiro & 17 & 27,0 \\
\hline Técnico de enfermagem & 26 & 41,3 \\
\hline Farmacêutico & 4 & 6,3 \\
\hline Fisioterapeuta & 10 & 15,9 \\
\hline Médico & 4 & 6,3 \\
\hline Nutricionista & 1 & 1,6 \\
\hline Psicóloga & 1 & 1,6 \\
\hline \multicolumn{3}{|c|}{ Utiliza a Internet para pesquisa estudo e atualização } \\
\hline Sim & 61 & 96,8 \\
\hline Não & 2 & 3,2 \\
\hline \multicolumn{3}{|c|}{ Níveis de importância da Internet na pesquisa, estudo e atualização } \\
\hline 1 & 4 & 6,3 \\
\hline 2 & 0 & 0,0 \\
\hline 3 & 8 & 12,7 \\
\hline 4 & 10 & 15,9 \\
\hline 5 & 41 & 65,1 \\
\hline \multicolumn{3}{|l|}{ Frequência da pesquisa pela Internet } \\
\hline Diária & 40 & 63,5 \\
\hline Semanal & 17 & 27,0 \\
\hline Eventual & 4 & 6,3 \\
\hline Nunca & 2 & 3,2 \\
\hline \multicolumn{3}{|l|}{ Utiliza vídeos informativos do Youtube } \\
\hline Sim & 56 & 88,9 \\
\hline Não & 7 & 11,1 \\
\hline \multicolumn{3}{|l|}{ Meio utilizado para buscar atualização } \\
\hline Banco de dados do próprio hospital & 3 & 4,8 \\
\hline E-books & 1 & 1,6 \\
\hline Livros na forma física & 6 & 9,5 \\
\hline Materiais na internet & 52 & 82,5 \\
\hline Telefone & 1 & 1,6 \\
\hline
\end{tabular}

Legenda: Banco de dados do próprio hospital: panfletos, livros, vídeos; Materiais na Internet: vídeos, artigos, estudos, protocolos, entre outros. Fonte: Souza PE, et al., 2021.

Referente ao regime de trabalho na UTI, 47 entrevistados afirmaram atuar como plantonistas, e os demais, como servidores efetivos.

Entre os 47 plantonistas, 24 declararam assumir três plantões semanais e dois profissionais referiram realizar mais de dez plantões semanais (Gráfico 1). 
Gráfico 1 - Frequência absoluta da quantidade de plantões semanais realizados por intensivistas atuantes em UTI COVID, de Hospital Universitário, de 17 a 21 de agosto de 2020 (n=63).

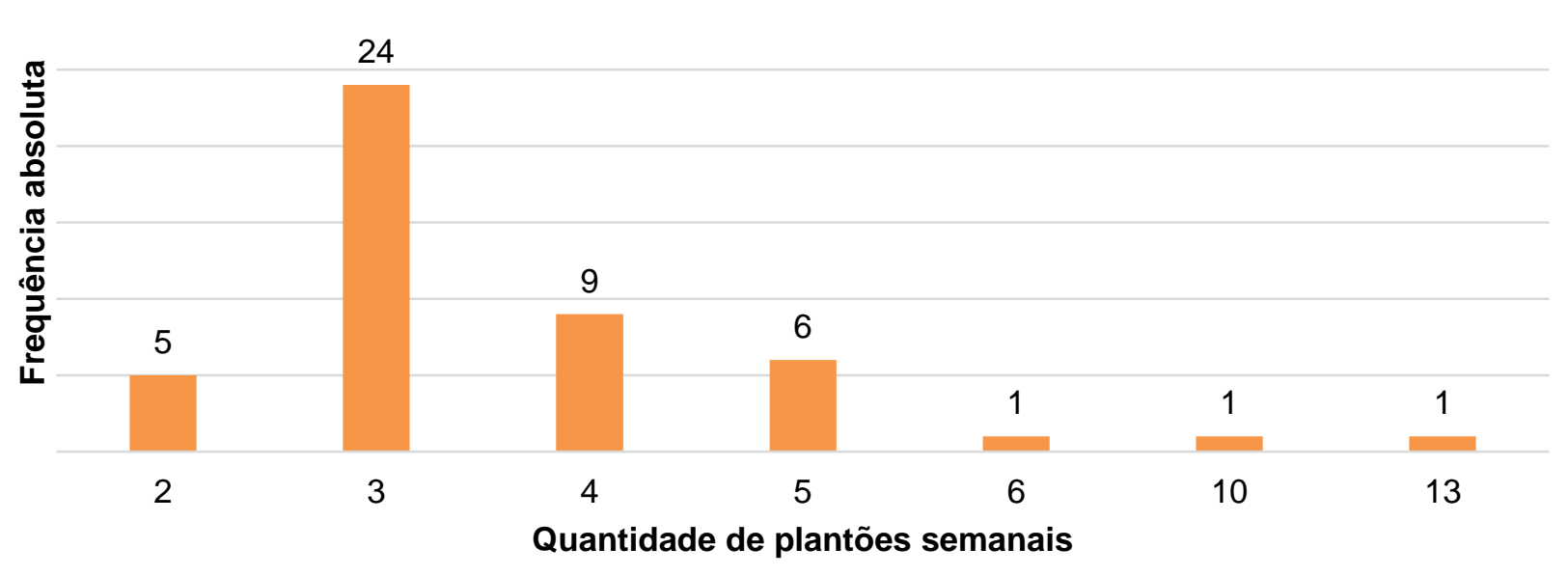

Fonte: Souza PE, et al., 2021

Independentemente do sistema de trabalho, 49 participantes referem uma carga de trabalho diária de 12 horas; 11, de seis horas; dois, de oito horas e um, desempenha suas atividades diárias em seis horas e meia.

Observando o tempo de atuação profissional pode-se inferir que 17 participantes possuem até sete anos de atuação; 30 profissionais atuam entre nove e 16 anos, e 16 já atuam há pelo menos 17 anos (Gráfico 2).

Gráfico 2 - Frequência absoluta do tempo de atuação profissional de intensivistas atuantes em UTI COVID, de Hospital Universitário, de 17 a 21 de agosto de 2020 ( $n=63)$.

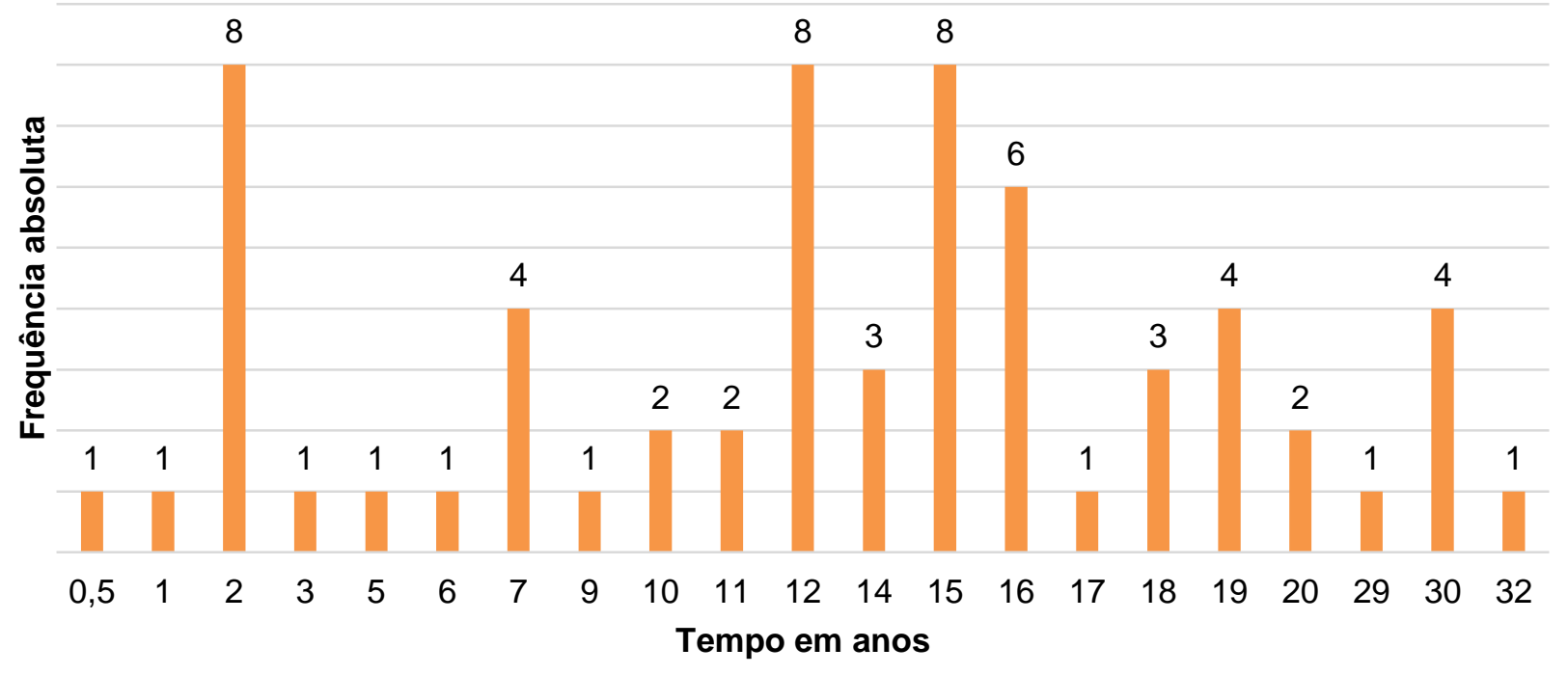

Fonte: Souza PE, et al., 2021.

Ao realizar abordagem com objetivo de comparar o tempo de realização das atividades na prática profissional diária, observou-se que, ao serem consultados sobre as atividades mais demoradas, a resposta mais prevalente referiu-se aos banhos e curativos dos pacientes $(42,9 \%)$, seguida da atividade de percorrer os leitos dos pacientes, inteirando-se da situação de cada um e dos cuidados necessários (25,0\%). Evoluir em prontuário, comunicação com colegas e burocracia apresentaram as menores frequências $(1,8 \%)$ (Gráfico 3 A). 
Quando avaliadas as atividades consideradas menos demoradas, as funções correlacionadas com menor duração foram a passagem de plantão entre os colegas $(30,4 \%)$ e movimentação do paciente no leito $(23,9 \%)$ (Gráfico 3 B).

Gráfico 3 - Atividades consideradas mais demoradas e menos demoradas, por intensivistas atuantes em UTI COVID, de Hospital Universitário, de 17 a 21 de agosto de $2020(n=63)$.

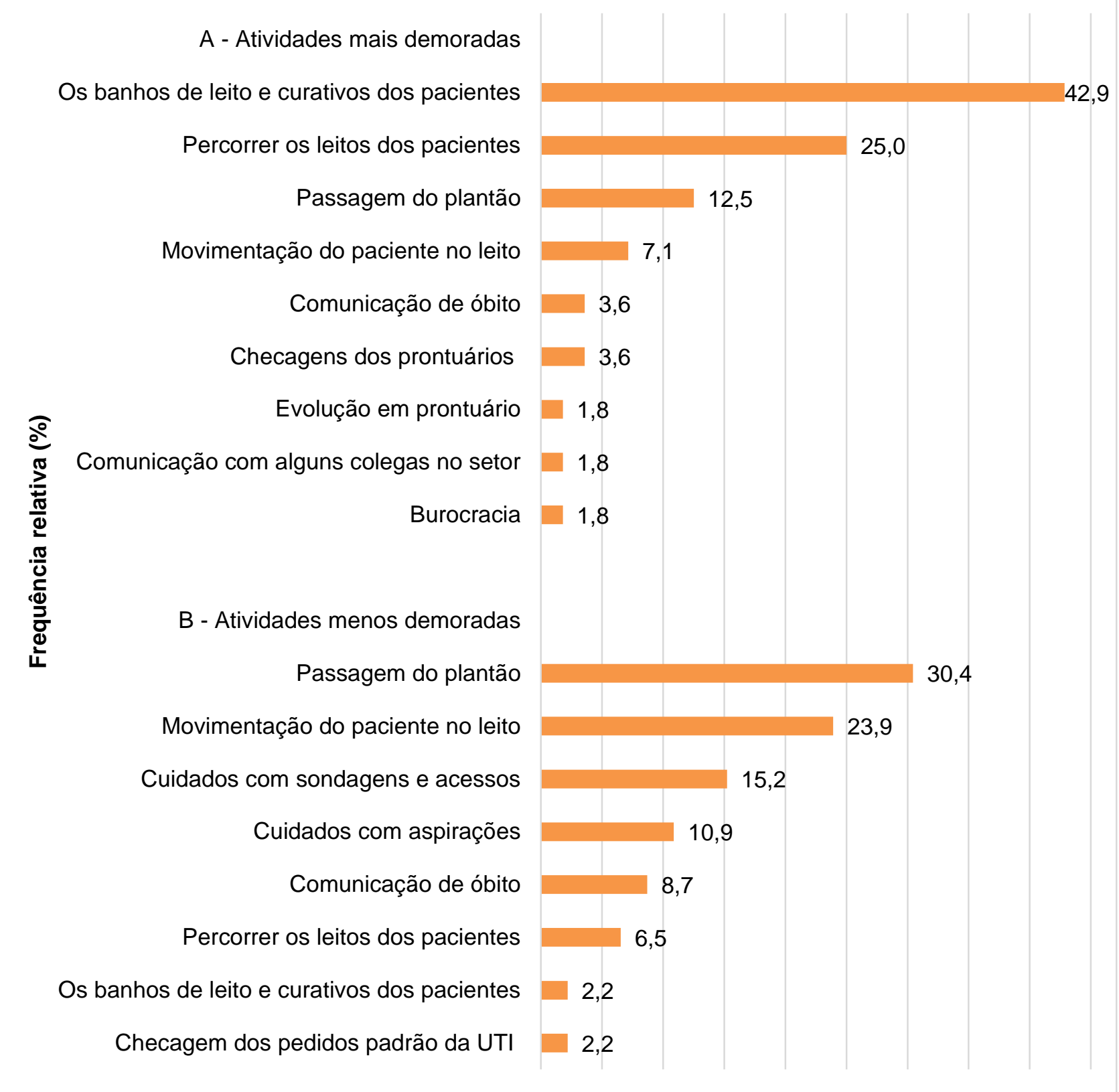

Legenda: Percorrer os leitos dos pacientes: percorrer os leitos dos pacientes, inteirando-se da situação de cada um e dos cuidados que necessita; Passagem do plantão: passagem do plantão com o relato das principais ocorrências do período; Cuidados com aspirações: cuidados com aspirações de traqueostomia e sonda nasogástrica. Sete profissionais não responderam à pergunta sobre a atividade mais demorada e 17, à questão sobre a função menos demorada. Fonte: Souza PE, et al., 2021

Realizada a comparação acerca do cansaço produzido pelas diferentes atividades, observou-se, que as atividades que foram citadas como mais cansativas entre os profissionais, foi a realização de banhos no leito e curativos (26,5\%) e percorrer os leitos dos pacientes (24,5\%) (Gráfico 4 A). 
A função menos cansativa, que apresentou a maior frequência, foi a passagem do plantão entre os colegas $(72,9 \%)$ (Gráfico 4 B).

Gráfico 4 - Atividades consideradas mais cansativas e menos cansativas, por intensivistas atuantes em UTI COVID, de Hospital Universitário, de 17 a 21 de agosto de 2020 ( $n=63)$.

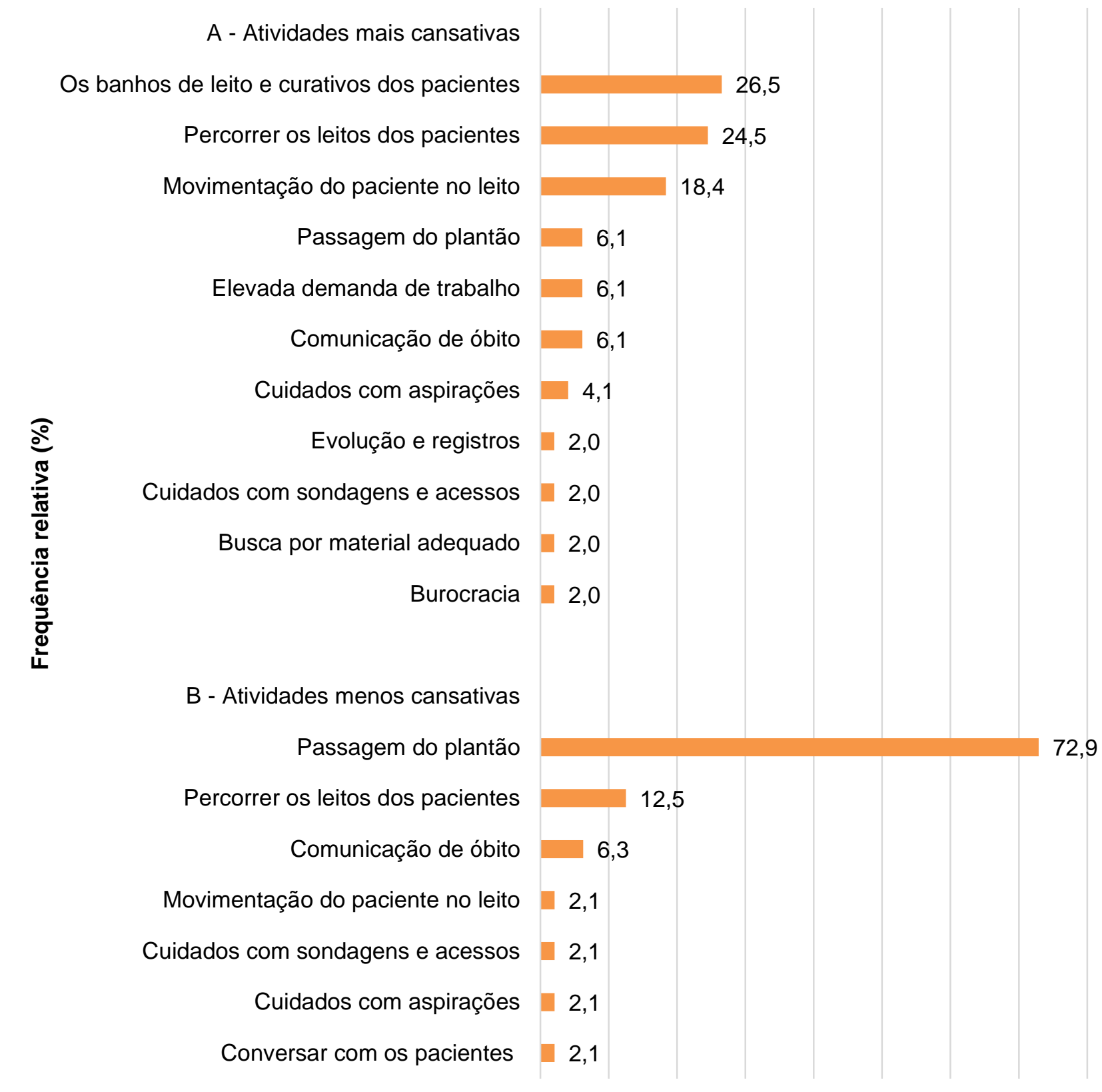

Legenda: Percorrer os leitos dos pacientes: percorrer os leitos dos pacientes, inteirando-se da situação de cada um e dos cuidados que necessita; Passagem do plantão: passagem do plantão com o relato das principais ocorrências do período; Cuidados com aspirações: cuidados com aspirações de traqueostomia e sonda nasogástrica. Catorze profissionais não responderam à pergunta sobre a atividade mais cansativa e 15, à questão sobre a função menos cansativa. Fonte: Souza PE, et al., 2021.

Entre os participantes, 46\% apresentaram teste positivo para COVID-19. Referindo-se às principais ações para aumento da segurança dos profissionais, em relação à infecção por COVID-19, mais frequentemente foi destacado que a melhor opção seria a realização de treinamentos permanentes sobre COVID-19 e outras doenças infectocontagiosas (41,3\%) (Gráfico 5). 
Gráfico 5 - Frequência relativa de ações para combate de infecções por COVID-19 desenvolvidas por intensivistas atuantes em UTI COVID, de Hospital Universitário, de 17 a 21 de agosto de 2020 (n=63).

Cuidados permanentes no manuseio de pacientes COVID positivo

\section{Explicação frequente de EPIs}

Normas de conduta e procedimentos que o profissional deve ter com a própria saúde

Programas de educação permanente para controle de infecções hospitalares

Treinamento específico e permanente sobre o covid e outras doenças infecciosas

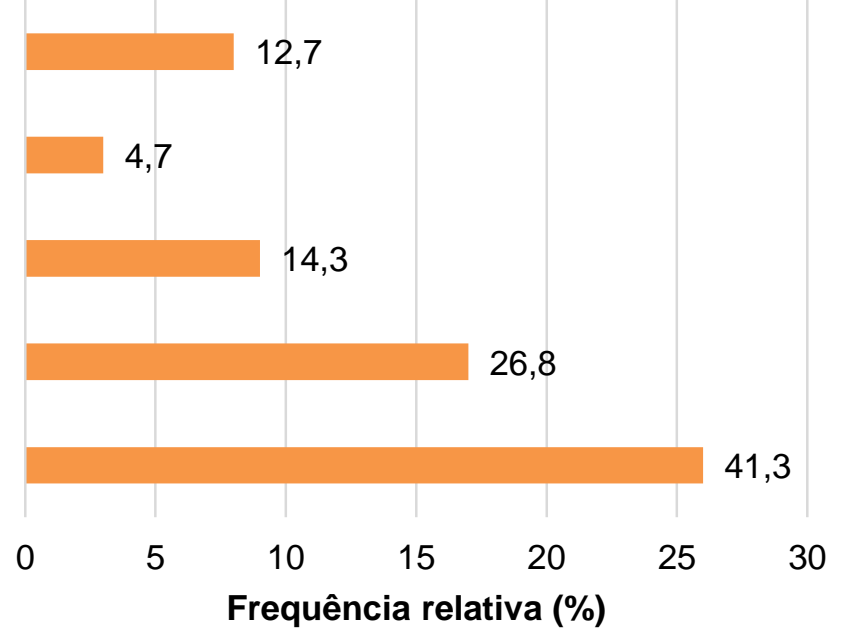

Fonte: Souza PE, et al., 2021.

\section{DISCUSSÃO}

A amostra apresentou prevalência da faixa etária entre 31 e 40 anos e $52,4 \%$ dos indivíduos possuíam menos de 40 anos, perfil etário semelhante ao de outras equipes intensivistas do país (ALVES FAD, et al., 2020; SANTOS SAA, et al., 2021; HASSEGAWA LCU, et al., 2017; MARQUES GLC, et al., 2018).

Os profissionais enfermeiros, técnicos de enfermagem e fisioterapeutas compuseram a maior parte dos trabalhadores da equipe de UTI, o que também é evidenciado em outras unidades (OLIVEIRA VPS e SILVA HR, 2021; RODRIGUES MA, et al., 2017).

A distribuição de equipes de UTI varia entre os serviços, de acordo com as demandas do atendimento prestado. Dependendo da atuação, apresenta-se com características de composição profissional e de processos diferenciados, nos quais as atribuições profissionais da equipe podem diferir daquelas solicitadas por unidades com pacientes COVID. (CONSELHO FEDERAL DE MEDICINA, 2020).

As unidades dedicadas exclusivamente para o atendimento de pacientes COVID, como a descrita no presente estudo, abordam casos de uma doença grave que ainda não foi completamente descrita, inviabilizando a existência de diretrizes ou padronizações completamente eficientes, fatores que incialmente justificam a composição da equipe apresentada (ORGANIZAÇÃO PAN-AMERICANA DA SAÚDE, 2020).

O perfil da doença, associado à instabilidade hemodinâmica, exige monitorização constante da equipe, em conjunto com a realização de intervenções rápidas (PASCARELLA G, et al., 2020). Súplicas que, inevitavelmente, acrescentam quantidade de trabalho, documentações e procedimentos burocráticos, são fatores contributivos para o maior número de pessoal, como expressado. Há necessidade de estrutura especial e recursos humanos com habilidades e capacidades específicas e indispensáveis para atender aos pacientes de forma eficiente e efetiva (ORGANIZAÇÃO PAN-AMERICANA DA SAÚDE, 2020).

A equipe avaliada apresentou um tempo médio em torno de 13 anos de atuação profissional, considerado de bom, pois o tempo revela ser um marcador de experiência. Porém, apesar da complexidade do serviço intensivista, são relatadas várias equipes compostas por profissionais recém-formados (HASSEGAWA LCU, et al., 2017; MARQUES GLC, et al., 2018). A justificativa para tal situação é proveniente da escassez de indivíduos especializados, fato que ocorre tanto no Brasil quanto nos Estados Unidos da América (HASSEGAWA LCU, et al., 2017; PASTORES SM e KVETAN V, 2015).

Dentro deste contexto, o desenvolvimento contínuo do profissional de saúde traz benefícios, motivando-o nos desafios diários da profissão. Esses benefícios são advindos de melhoras nos aspectos cognitivos, mentais e emocionais (DRUDE KP, et al., 2019). Para o aprimoramento, o tempo é crucial, e o 
conhecimento adquirido redundará em maior competência clínica e performance profissional. Assim, em geral, profissionais com maior experiência (e consequentemente maior idade) estão mais aptos para enfrentar as adversidades do atendimento a pacientes graves, hospitalizados por COVID, especialmente nos primórdios dessa afecção (STEVENSON R e MOORE D, 2018).

Em que pese, a idade elevada se apresentou como um dos principais fatores de gravidade e mortalidade para COVID-19 (CHEN T, et al., 2020). Esta condição causou a morte de milhares de profissionais de saúde brasileiros, culminando no afastamento de profissionais com mais idade, do ambiente hospitalar (MEDEIROS EAS, 2020).

Essa preocupação se alterou com o surgimento de variantes do Sars-CoV-2, que passaram a acometer com gravidade, também, indivíduos jovens (ORGANIZAÇÃO PAN-AMERICANA DA SAÚDE, 2020). Este fato gerou modificações dos critérios de suscetibilidade do profissional, influindo nas características da composição da equipe.

Nesse cenário, a vacinação contra a COVID-19 minimizou tais dicotomias, porque trouxe menor suscetibilidade à infecção, associada à recuperação precoce, diminuindo a severidade da doença e permitindo que equipes mais experientes como a apresentada possam atuar, com maior tranquilidade, dentro das margens de contaminação hospitalar (PALTIEL AD, et al., 2021).

Em relação à saúde psicológica dos trabalhadores em UTI COVID, um expediente extenso é verificado como fator deflagrante de estresse e de síndrome de Burnout (MARQUES GLC, et al., 2018). Além de que a carga horária do profissional atuante em UTI apresenta uma relação inversa com a percepção de segurança por parte do paciente (RUIVO BARA, et al., 2020). Contudo, o regime de serviço apresentado, em sua maioria, por meio de três plantões semanais com carga horária de 12 horas, corresponde ao apresentado em outras equipes, reafirmando ser essa a atuação típica do profissional de saúde atuante no intensivismo (ALVES FAD, et al., 2020; MARQUES GLC, et al., 2018). Tamanha carga horária constitui-se, portanto, em fator estressor nessa classe de profissionais.

Ainda, o amplo contato que esses profissionais têm com os pacientes aumenta o apego emocional, colaborando com alterações emocionais, o que facilita gatilhos psicológicos de estresse (LEITE MR e MONTELO NMS, 2021; OLIVEIRA VPS e SILVA HR, 2021).

A descrição das atividades mais cansativas foi coerente com as atividades mais demoradas. Na literatura está evidenciado que a exaustão ou o esgotamento dos trabalhadores frente à intensa carga de trabalho associa-se à ansiedade, sugerindo que o tempo para execução das tarefas faz um paralelismo com o estresse por elas gerado (TEIXEIRA CFS, et al., 2020).

Entre os participantes, 46\% foram acometidos por COVID-19, frequência elevada, quando comparada com outro hospital universitário brasileiro que apresentou 11,5\% de infecções entre os profissionais de UTI COVID (ESCUDERO DVS, et al., 2021). E, sem dúvida, esta ocorrência torna-se mais um fator desencadeador de alterações na saúde mental (ARSLAN G, et al., 2020).

Frente à taxa de contágio, os participantes sugeriram medidas para evitar a contaminação, como a realização de capacitações da equipe a respeito do controle de doenças infecciosas, propostas que confluem com os protocolos nacionais e que devem ocorrer de forma rápida, demonstrando comprometimento dos gestores com um ambiente seguro de trabalho (TEIXEIRA CFS, et al., 2020).

Além da capacitação de profissionais, a prevenção de infecções (não apenas por Sars-CoV-2) requer uma estrutura hospitalar organizada e dinâmica, permitindo flexibilidade para tomada de decisões imediatas (LIU Y, et al., 2020). A dinâmica hospitalar para prevenção deve, inicialmente, realizar barreiras de contato entre os infectados e profissionais que não atuam diretamente com o paciente (GALLASCH CH, et al., 2020). Atitudes estas tomadas no hospital em que a pesquisa foi desenvolvida, separando os setores administrativos dos demais. Dentre os profissionais que atuam em contato com pacientes contaminados é fundamental a utilização adequada de EPIs (NATIONAL CENTER FOR CHRONIC DISEASE PREVENTION AND HEALTH PROMOTION, 2019). Apesar do exposto inúmeros serviços, segundo revisão realizada, apresentaram falta do aporte adequado de EPIs, dificultando a correta proteção dos servidores (TEIXEIRA CFS, et al., 2020). 
A instalação de serviços exclusivos para pacientes infectados e o cancelamento de procedimentos não urgentes, diminuem os riscos de infecção, pois diminuem o fluxo de pessoas e direcionam os contaminados aos locais mais bem estruturados e preparados neste sentido (GALLASCH CH, et al., 2020). Estas providências foram desenvolvidas no serviço analisado, limitando os atendimentos e as intervenções aqueles considerados imprescindíveis.

As capacitações corriqueiras dos profissionais favorecem a atualização e elucidação de dúvidas. A maioria dos participantes afirmou usar a Internet para sanar dúvidas relacionadas ao serviço e, da mesma forma, atestou que a Internet tem elevada importância no trabalho, sendo a principal forma de atualização utilizada. Estes resultados evidenciam que o uso da Internet é uma opção viável para o treinamento na saúde, além de facilitar o acesso ao ensino, uma vez que permite a conectividade a partir de qualquer local, moldando a tecnologia às necessidades do usuário (NICOLL P, et al., 2018).

Outra vantagem do ensino online é a oportunidade de capacitação simultânea de profissionais em serviço, possibilitando conferências entre um número expressivo de pessoas, mesmo sem estrutura física para acomodar tamanho contingente (MORAN JM, 2003). Um benefício que permite a evolução síncrona de todo grupo sem gerar aglomerações, mantendo as normas de biossegurança impostas.

Ao abordar o ensino online durante o período pandêmico são destacáveis as diferenças entre a educação online e o ensino remoto emergencial. A educação online, já consolidada entre instituições de ensino, conta com suporte técnico associado a um curso, embasado em metodologias de ensino remotas. Características diferentes das apresentadas no ensino remoto de emergência, que tem como objetivo suprir uma demanda momentânea, que exige adaptações ou providências não disponíveis (HODGES C, et al., 2020).

A respeito da natureza do conteúdo visualizado na internet, a principal escolha de informação foram os vídeos no Youtube, plataforma que já disponibiliza conteúdo médico de fácil acesso, contudo que ainda carece de maior rigor metodológico para validação das informações disponibilizadas (YUKSEL B e CAKMAK K, 2020). A pesquisa no Youtube apresentou maior expressão de interatividade por parte dos consultados, associada a feedbacks positivos, sugerindo que o aprendizado online já é uma realidade (QUINTANILHA LF, 2017).

O presente estudo realizou uma investigação que, embora ocorrida em curto espaço de tempo, trouxe informações, ainda não muito discutidas na literatura, em virtude de a pandemia ser um evento recente e, assim, contribuições para elucidação de seus aspectos são muito importantes.

\section{CONCLUSÃO}

Os resultados demonstraram que a UTI COVID-19 do Hospital Universitário analisado foi composta, majoritariamente, por enfermeiros e técnicos de enfermagem com idade inferior a 40 anos, que trabalhavam, principalmente, em regime de plantões de 12 horas, em média três vezes na semana. A alta taxa de infecção entre os membros da equipe, a demora e o cansaço na realização de determinadas atividades, bem como o regime de trabalho sugerem ser fatores estressores na equipe. A Internet se mostrou o principal meio de atualização utilizado, especialmente por meio de vídeo aulas.

\section{REFERÊNCIAS}

1. ALVES FAD, et al. Perfil dos fisioterapeutas nas unidades de terapia intensiva adulto. Revista Eletrônica Acervo Saúde, 2020; sup55: 1-11.

2. ARSLAN G, et al. Coronavirus Stress, Optimism-Pessimism, Psychological Inflexibility, and Psychological Health: Psychometric Properties of the Coronavirus Stress Measure. International Journal of Mental Health and Addiction, 2020: 1-17.

3. BRASIL. Ministério da Saúde. MS-SUS COVID-19 Leitos. 2020. Disponível em: <https://qsprod.saude.gov.br/extensions/Leitos_C19/Leitos_C19.html>. Acessado em: 22 de março de 2021.

4. BROOKS SK, et al. The psychological impact of quarantine and how to reduce it: rapid review of the evidence. The Lancet, 2020; 395(10227): 912-920.

5. CHEN T, et al. Clinical characteristics of 113 deceased patients with coronavirus disease 2019: retrospective study. British Medical Journal, 2020; 368: m1091. 
6. CONSELHO FEDERAL DE MEDICINA. Resolução No 2271, de 14 de fevereiro de 2020. Disponível em: https://www.in.gov.br/en/web/dou/-/resolucao-n-2.271-de-14-de-fevereiro-de-2020-253606068. Acessado em: 3 de julho de 2021.

7. DRUDE KP, et al. Continuing Professional Development. Reflections on a Lifelong Learning Process. Psychiatric Clinics of North America, 2019; 42(3): 447-461.

8. ESCUDERO DVS, et al. Prevalência de SARS-COV-2 entre profissionais da saúde de um hospital terciário de ensino. The Brazilian Journal of Infectious Diseases, 2021; 25(S1).

9. FORTALEZA CMCB. Evidence, rationality, and ignorance: Agnotological issues in COVID-19 science. Revista da Sociedade Brasileira de Medicina Tropical Online, 2020; 53(e20200475): 1-5.

10. GALLASCH CH, et al. Prevenção relacionada à exposição ocupacional do profissional de saúde no cenário de COVID-19. Revista Enfermagem UERJ, 2020; 28: e49596.

11. HASSEGAWA LCU, et al. Perfil da Formação Médica em Terapia Intensiva no Estado de Rondônia. Revista Brasileira de Educação Médica, 2017; 41(1): 38-43.

12. HODGES C, et al. As diferenças entre o aprendizado online e o ensino remoto de emergência. Revista da Escola, Professor, Educação e Tecnologia, 2020; 2.

13. LEITE MR, MONTELO NMS. Profissionais de saúde e sua relação com a morte e o morrer de pacientes em UTI. Revista Eletrônica Acervo Saúde, 2021; 13(2): 1-8.

14. LIU Y, et al. Critical care response to a hospital outbreak of the $2019-n C o V$ infection in Shenzhen, China. Critical Care, 2020; 24(56).

15. MARQUES GLC, et al. Síndrome de burnout entre médicos plantonistas de unidades de terapia intensiva. Jornal Brasileiro de Psiquiatria, 2018; 67(3):186-93.

16. MARTINS BG, et al. Escala de Depressão, Ansiedade e Estresse: propriedades psicométricas e prevalência das afetividades. Jornal Brasileiro de Psiquiatria, 2019; 68(1): 32-41.

17. MASLACH C, LEITER MP. Understanding the burnout experience: recent research and its implications for psychiatry. World Psychiatry, 2016; 15(2): 103-111.

18. MEDEIROS EAS. A luta dos profissionais de saúde no enfrentamento da COVID-19. Acta Paulista de Enfermagem, 2020; 33:e-EDT20200003.

19. MORAN JM. Contribuições para uma pedagogia da educação a distância no ensino superior. Interface Comunicação, Saúde, Educação, 2003; 7(12): 139-48.

20. MORGANTINI LA, et al. Factors contributing to healthcare professional burnout during the COVID-19 pandemic: A rapid turnaround global survey. PLOS ONE, 2020; 15(9): e0238217.

21. MOURA A, et al. Fatores associados à ansiedade entre profissionais da atenção básica. Revista Portuguesa de Enfermagem de Saúde Mental, 2018; 19: 16-26.

22. NATIONAL CENTER FOR CHRONIC DISEASE PREVENTION AND HEALTH PROMOTION. Interim infection prevention and control recommendations for patients with suspected or confirmed coronavirus disease 2019 (COVID19) in healthcare settings. National Center for Chronic Disease Prevention and Health Promotion. Division of Diabetes, Translation (Atlanta, GA, 2020). Disponível em: https://stacks. cdc. gov/view/cdc/86043], Acessado em: 1 de julho de 2021.

23. NICOLL $P$, et al. Evaluation of technology-enhanced learning programs for health care professionals: Systematic review. Journal of Medical Internet Research, 2018; 20(4): e131.

24. OLIVEIRA VPS, SILVA HR. Prevalência da síndrome de burnout entre profissionais de saúde que atuam em unidades de terapia intensiva. Brazilian Journal of Development, 2021; 7(2): 17863-17875.

25. ORGANIZAÇÃO PAN-AMERICANA DA SAÚDE. Checklist para a gestão dos recursos humanos em saúde em resposta à COVID-19, 2020. Disponível em: <https://iris.paho.org/handle/10665.2/52158>.Acessado em: 2 de julho de 2021.

26. PALTIEL AD, et al. Clinical Outcomes of A COVID-19 Vaccine: Implementation Over Efficacy. Health Affairs, 2021; 40(1).

27. PASCARELLA G, et al. COVID-19 diagnosis and management: a comprehensive review. Journal of Internal Medicine, 2020; 288(2): 192-206.

28. PASTORES SM, KVETAN V. Shortage of intensive care specialists in the United States: recent insights and proposed solutions. Revista Brasileira de Terapia Intensiva, 2015; 27(1): 5-6.

29. QUINTANILHA LF. Inovação pedagógica universitária mediada pelo Facebook e YouTube: uma experiência de ensino-aprendizagem direcionado à geração-Z. Educar em Revista, 2017; 65: 249-263.

30. RODRIGUES MA, et al. Divergências entre legislações do dimensionamento de Enfermagem em unidades de terapia intensiva. Enfermagem em Foco, 2017; 8(1): 12-16.

31. RUIVO BARA, et al. Assistência de enfermagem na segurança do paciente na UTI: uma revisão integrativa da literatura. Revista Eletrônica Acervo Enfermagem, 2020; 5: e5221.

32. SALLES NETO LL, et al. Forecast UTI: aplicativo para previsão de leitos de unidades de terapia intensiva no contexto da pandemia de COVID-19. Epidemiologia e Serviços de Saúde, 2020; 29(4): e2020391.

33. SANTOS SAA, et al. Riscos ocupacionais em profissionais de enfermagem de uma Unidade de Terapia Intensiva adulta, localizada em um município de Pernambuco. Revista Eletrônica Acervo Saúde, 2021; 13(2): 1-8.

34. SILVA AF, ROBAZZI MLCC. Alterações mentais em trabalhadores de unidades de terapia intensiva. SMAD Revista Eletrônica Saúde Mental Álcool e Drogas, 2019;15(3): 1-10.

35. STEVENSON R, MOORE DE. Ascent to the Summit of the CME Pyramid. JAMA, 2018; 319(6): 543-544.

36. TEIXEIRA CFST, et al. A saúde dos profissionais de saúde no enfrentamento da pandemia de Covid-19. Ciências \& Saúde Coletiva, 2020; 25(9): 3465-3474.

37. TORRES JDRV, et al. Fatores associados à fadiga por compaixão em profissionais de saúde, no contexto hospitalar: uma revisão na literatura. Temas em Saúde, 2018; 18(3): 178-194.

38. WORLD HEALTH ORGANIZATION. WHO Coronavirus (COVID-19) Dashboard. Disponível em: <https://covid19. who.int/table>. Acessado em: 23 de março de 2021.

39. YILDIRIM M, SOLMAZ F. COVID-19 burnout, COVID-19 stress, and resilience: Initial psychometric properties of COVID-19 Burnout Scale. Death Studies, 2020; :1-9.

40. YUKSEL B, CAKMAK K. Healthcare information on YouTube: Pregnancy and COVID-19. International Journal of Gynecology \& Obstetrics, 2020; 150(2): 189-193. 